\title{
New insights into the clinical characteristics and prognostic factors of pulmonary fungal infections from a retrospective study in Southwestern China
}

This article was published in the following Dove Press journal: Infection and Drug Resistance

\section{Li Peng' \\ Zhiping $\mathrm{Xu}^{2}$ \\ Zhenyu Huo' \\ Rui Long ${ }^{3}$ \\ Liang $\mathrm{Ma}^{4}$}

'Department of Respiratory Medicine, The First Affiliated Hospital of Chongqing Medical University, 2Department of Respiratory Medicine, The Fifth People's Hospital of Chongqing, ${ }^{3}$ Department of Pharmacy, The First Affiliated Hospital of Chongqing Medical University, Chongqing, People's Republic of China; ${ }^{4}$ Critical Care Medicine Department, Clinical Center, National Institutes of Health, Bethesda, MD, USA
Correspondence: Li Peng

Department of Respiratory Medicine,

The First Affiliated Hospital of Chongqing

Medical University, No I Youyi Road,

Yuzhong District, Chongqing 4000I6,

People's Republic of China

Tel +8623890I 2806

Email pliI228@I63.com
Background: Despite increasing incidence of pulmonary fungal infections (PFIs) worldwide, the clinical characteristics and prognostic factors remain poorly understood. The goal of this study was to investigate the clinical features, laboratory findings, and outcomes of hospitalized patients diagnosed with PFIs.

Methods: We retrospectively enrolled 123 patients at a university hospital in Southwestern China between February 2014 and May 2016, who were diagnosed with PFIs based on clinical presentations and laboratory tests including fungal culture and pathological examination. Medical records were reviewed and analyzed. Prognostic factor associated with mortality was evaluated by multivariate regression analysis.

Results: Of the 123 PFI patients enrolled, the mean age was 67 years with $72 \%$ of them being males. In addition to common clinical features reported previously, these patients exhibited distinct characteristics, with the elderly accounting for $79 \%$ of all cases, and with prolonged hospitalization being the most prevalent risk factor (74\%) and chronic obstructive pulmonary disease (COPD) being the most common underlying disease (45\%). Invasive operation was significantly more frequently involved in patients with unfavorable treatment responses than in patients with favorable responses ( 45.6 vs $7.4 \%, P=0.000$ ). By multivariate regression analysis, invasive operation (odds ratio [OR]: 5.736, 95\% confidence interval [CI]: 2.008-16.389, $P=0.001$ ) and hypoalbuminemia (OR: $3.936,95 \%$ CI: $1.325-11.696, P=0.014$ ) were independent prognostic factors of mortality in PFIs.

Conclusion: This study provides new insights into the clinical characteristics and prognostic factors of PFIs and highlights the necessity to be aware of PFIs in patients with COPD and patients receiving invasive operation in order to improve clinical management of these patients.

Keywords: pulmonary fungal infection, risk factors, prognostic factors, chronic obstructive pulmonary disease, invasive operation

\section{Introduction}

With the advent of human immunodeficiency virus (HIV) epidemic and the everincreasing use of broad-spectrum antibiotics, glucocorticoids, immunosuppressive agents, and various types of invasive procedures, there has been a dramatic increase in the incidence of systemic or deep mycoses. ${ }^{1}$ Susceptible individuals are usually infected by the inhalation of fungal spores into the respiratory tract, initiating a pulmonary fungal infection (PFI) and subsequently spreading to other organs. ${ }^{2}$ In most cases, the initial pulmonary infection is mild or subclinical. The clinical manifestations and imaging characteristics of PFIs are often not typical or specific and thus can be easily overlooked or misdiagnosed by clinician. In fact, currently, laboratory diagnosis 
of PFIs is also challenging. Although antigen and antibody tests are available for clinical use, the definitive diagnosis of PFIs relies primarily on in vitro fungal culture and/or microscopic examination of respiratory specimens, which are usually time-consuming or lack sensitivity, thereby leading to a delayed or missed diagnosis. ${ }^{1,3}$

As a result of the challenges earlier, many aspects of PFIs, including clinical characteristics and prognostic factor, remain poorly understood. Most of the reported studies on PFIs have focused on special population with a selected condition or infected with a particular fungal species, particularly Aspergillus spp. There are only sparse reports on PFIs in patients with a wide range of risk factors and clinical background. ${ }^{4-6}$ In the present study, we retrospectively investigated a case series of PFI patients with diverse demographic factors and medical conditions and demonstrated their clinical characteristics as well as treatment outcomes.

\section{Methods}

\section{PFI case definition}

We reviewed the medical records of all hospitalized patients diagnosed with PFIs from February 2014 to May 2016 in the Department of Respiratory and Critical Care Medicine, a 3000-bed teaching hospital in southwestern China. PFI diagnosis and classification were established according to the revised definitions of invasive fungal disease. ${ }^{7}$ Diagnostic criteria included 1 ) at least one of the 22 previously identified risk factors (Table 1); ;, ${ }^{12} 2$ 2) at least one of the symptoms including cough, expectoration, fever, hemoptysis, chest pain, and dyspnea; 3) positive chest radiological findings including halo sign, air crescent sign, macronodule $(\geq 1 \mathrm{~cm}$ in diameter), nodule, consolidation, cavity lesion, air bronchograms or bronchiectasis; ${ }^{13} 4$ ) elevated serum 1,3- $\beta$-D-glucan level $(>60 \mathrm{pg} / \mathrm{mL})$ or identification of fungal species in the culture of blood sample (positive once) or respiratory samples (positive twice in culture of sputum or once in culture of pleural fluid, transtracheal aspirate, percutaneous needle aspiration biopsy, or bronchoalveolar lavage fluid); and 5) positive microscopic examination of computed tomography (CT)-guided percutaneous needle aspiration biopsy of the lung. PFI cases were classified into "possible", "probable", and "proven". Possible PFI was defined if the patient fulfilled criteria 1, 2, and 3; probable PFI was defined if the patient fulfilled criteria 1, 2, 3, and 4; and proven PFI was defined if the patient fulfilled all the five criteria. Excluded were cases with incomplete clinical data, no radiological data, or prophylactic use of antifungal drugs.
Table I Prevalence of risk factors in 123 patients with PFIs

\begin{tabular}{|c|c|c|}
\hline Risk factors & $\begin{array}{l}\text { Number of } \\
\text { patients }\end{array}$ & $\%$ \\
\hline Prolonged hospitalization ( $>10$ days) & 91 & 73.9 \\
\hline Smoking & 66 & 53.6 \\
\hline $\begin{array}{l}\text { Prolonged use of broad-spectrum antibiotics } \\
\text { ( }>2 \text { weeks) }\end{array}$ & 60 & 48.7 \\
\hline Chronic obstructive pulmonary disease & 55 & 44.7 \\
\hline Invasive operation & $30^{\mathrm{a}}$ & 24.3 \\
\hline Hypoalbuminemia $(<30 \mathrm{~g} / \mathrm{L})$ & 30 & 24.3 \\
\hline Hematological or solid organ malignancy & 20 & 16.2 \\
\hline $\begin{array}{l}\text { Prolonged high-dose corticosteroid treatment } \\
\text { in previous } 60 \text { days ( }>3 \text { weeks) }\end{array}$ & 18 & 13.5 \\
\hline Other pulmonary structural disorders & 18 & 13.5 \\
\hline Diabetes mellitus & 14 & 11.3 \\
\hline Chronic renal disorder & 10 & 7.5 \\
\hline Systemic inflammatory diseases & 8 & 6.5 \\
\hline Invasive fungal infection in previous 2 years & 7 & 5.6 \\
\hline $\begin{array}{l}\text { Use of immunosuppression agents in previous } \\
30 \text { days }\end{array}$ & 5 & 3.7 \\
\hline Advanced HIVIAIDS & 3 & 2.4 \\
\hline $\begin{array}{l}\text { Significant neutropenia for }>10 \text { days in previous } \\
60 \text { days }\end{array}$ & 2 & 1.6 \\
\hline $\begin{array}{l}\text { Bone marrow transplantation or solid organ } \\
\text { transplantation }\end{array}$ & 2 & 1.6 \\
\hline $\begin{array}{l}\text { Primary immune deficiency affecting neutrophil } \\
\text { function }\end{array}$ & 0 & 0 \\
\hline History of sinus disease & 0 & 0 \\
\hline Prolonged granulocytopenia (>3 weeks) & 0 & 0 \\
\hline Chronic granulomatous disease & 0 & 0 \\
\hline Liver cirrhosis & 0 & 0 \\
\hline
\end{tabular}

Note: all received urinary catheterization for 2-30 days, with five of them also receiving invasive mechanical ventilation for 4-12 days.

Abbreviations: AIDS, acquired immune deficiency syndrome; HIV, human immunodeficiency virus; PFIs, pulmonary fungal infections.

\section{Clinical data collection}

For all patients, we retrieved medical data, including potential risk factors (Table 1), clinical presentations and laboratory findings (Tables 2 and 3), and treatment and outcomes (Table 4). All patients were treated with voriconazole, itraconazole, fluconazole, caspofungin, or amphotericin B (Table 4). The changes of symptoms, signs, and laboratory tests during and after treatment were recorded. None of these patients received surgical resection, growth factors, granulocyte transfusions, or other adjuvant therapies. Treatment outcomes included favorable and unfavorable responses during hospitalization. Favorable response was defined as a reduction of more than half of the symptoms and rale signs with or without a partial or complete resolution of pulmonary lesions observed before treatment. Unfavorable response was defined as worsening, or no symptomatic or radiological improvement, or death during hospitalization regardless of the cause. ${ }^{14}$ 
Table 2 Clinical characteristics of 123 patients with PFIs

\begin{tabular}{|c|c|c|}
\hline Characteristics & $\begin{array}{l}\text { Number of } \\
\text { patients }\end{array}$ & $\%$ \\
\hline \multicolumn{3}{|l|}{$\mathrm{Age}^{\mathrm{a}}$} \\
\hline Children (0-9 years) & 0 & 0 \\
\hline Adolescent and adults ( $10-59$ years) & 26 & 21.1 \\
\hline Elderly ( $\geq 60$ years) & 97 & 78.9 \\
\hline Gender (male) & 89 & 72.4 \\
\hline Current or exsmoker & 66 & 53.6 \\
\hline Current or former alcohol drinker & 43 & 34.9 \\
\hline \multicolumn{3}{|l|}{ Pulmonary or systemic symptoms and signs } \\
\hline Cough and expectoration & 113 & 91.8 \\
\hline Fever & 47 & 38.2 \\
\hline Dyspnea & 25 & 20.3 \\
\hline Chest pain & 14 & 11.3 \\
\hline Hemoptysis & 8 & 6.5 \\
\hline Abnormal chest signs & 103 & 83.7 \\
\hline Oral or skin fungal infection signs & 42 & 34.1 \\
\hline \multicolumn{3}{|l|}{ Chest imaging findings } \\
\hline Cavity lesions & 11 & 8.9 \\
\hline Nodules & 30 & 24.4 \\
\hline Pleural effusion & 26 & 21.1 \\
\hline \multicolumn{3}{|l|}{ Underlying or accompanying diseases } \\
\hline Chronic obstructive pulmonary disease & 55 & 44.7 \\
\hline Hypertension & 31 & 25.2 \\
\hline Heart disease & 27 & 22.0 \\
\hline Malignant tumor & 20 & 16.3 \\
\hline Diabetes mellitus & 14 & 11.3 \\
\hline Chronic renal disorder & 10 & 8.1 \\
\hline Bronchiectasis & 9 & 7.3 \\
\hline Interstitial lung disease & 6 & 4.9 \\
\hline Tuberculosis & 6 & 4.9 \\
\hline Asthma & 4 & 3.3 \\
\hline Pneumonia & 4 & 3.3 \\
\hline Dermatomyositis & 3 & 2.4 \\
\hline HIV infection & 3 & 2.4 \\
\hline Renal transplantation & 2 & 1.6 \\
\hline Systemic lupus erythematosus & 2 & 1.6 \\
\hline
\end{tabular}

Notes: ${ }^{a}$ Classified according to the latest criteria of the World Health Organization. ${ }^{36}$ The adolescent and adult groups were combined due to the small number of adolescents (only one).

Abbreviations: HIV, human immunodeficiency virus; PFI, pulmonary fungal infection.

\section{Statistical analysis}

Categorical variables were presented as numbers (percentages) and analyzed by Chi-square test or Fisher's exact test, while continuous variables were presented as medians and analyzed by Mann-Whitney test or $t$ test. Multivariate logistic regression analysis was used to analyze the association between risk factors and mortality with IBM SPSS Statistics 22. Other statistical analyses were performed using GraphPad Prism 5.01 (GraphPad Software, Inc., La Jolla, CA, USA). A two side $P<0.05$ was considered statistically significant.
Table 3 Fungus culture and I,3- $\beta$-D-glucan test in 60 patients with probable/proven PFIs

\begin{tabular}{lll}
\hline Fungus examination & Number of patients & $\%$ \\
\hline I,3- $\beta$-D-Glucan test & & \\
Positive $(\geq 60 \mathrm{pg} / \mathrm{mL})$ & 44 & 73.3 \\
Negative $(<60 \mathrm{pg} / \mathrm{mL})$ & 16 & 26.7 \\
Positive fungal culture & $39^{\mathrm{a}}$ & 65.0 \\
Candida albicans & $20^{\mathrm{b}}$ & $51.3^{\mathrm{d}}$ \\
Candida tropicalis & 3 & $7.6^{\mathrm{d}}$ \\
Candida glabrata & 2 & $5.1^{\mathrm{d}}$ \\
Candida klebsiella & $\mathrm{I}$ & $2.6^{\mathrm{d}}$ \\
Aspergillus fumigatus & $10^{\mathrm{c}}$ & $25.6^{\mathrm{d}}$ \\
Aspergillus niger & 1 & $2.6^{\mathrm{d}}$ \\
Cryptococcus & 2 & $5.1^{\mathrm{d}}$ \\
Negative fungal culture & 21 & 35.0 \\
\hline
\end{tabular}

Notes: a Confirmed by fungal culture and/or pathological examination. 'Including eight from bronchoalveolar lavage fluids, two from pleural fluids, and 10 from lower respiratory tract secretions. Including two from percutaneous needle aspiration biopsies, four from bronchoalveolar lavage fluids, and four from lower respiratory tract secretions. ${ }^{d}$ Relative to the total number (39) of patients positive in fungal culture. Abbreviation: PFI, pulmonary fungal infection.

\section{Institutional review board statement}

This study was approved by Institutional Review Board of the First Affiliated Hospital of Chongqing Medical University (no 20171801). Written informed consent was deemed unnecessary by the Institutional Review Boards for human studies due to the retrospective nature of the data. All patient data were de-identified and treated with the utmost confidentiality in accordance with Institutional Review Board requirements.

\section{Results \\ Risk factors for PFls}

We identified a total of 123 patients with PFIs and evaluated the prevalence of risk factors known to be associated with PFIs (Table 1) ${ }^{1,9-12}$ The most common risk factor was prolonged hospitalization (>10 days), which was noted in $74 \%$ of patients. Other common risk factors included smoking (54\%), prolonged use of broad-spectrum antibiotics (48\%), and chronic obstructive pulmonary disease (COPD; 45\%). Relatively uncommon risk factors included invasive operation (24\%), hypoalbuminemia (24\%), hematological or solid organ malignancy (16\%), high-dose corticosteroid treatment (14\%), pulmonary structural disorder (14\%), and diabetes mellitus $(11 \%)$. All the remaining risk factors are rare (each $<10 \%)$.

\section{Clinical characteristics of PFIs}

Of the 123 PFI patients enrolled, all were native Chinese from the Southwest of China and had a mean age of 67 years (ranging $16-91$ years), with $79 \%$ of them being elderly ( $\geq 60$ years), 
Table 4 Comparison of characteristics between patients with favorable responses and those with unfavorable responses

\begin{tabular}{|c|c|c|c|}
\hline Characteristics & Favorable response $^{a}(n=68)$ & Unfavorable response $^{a}(n=55)$ & $P$-value ${ }^{b}$ \\
\hline Age (years) & $66.5 \pm 13.5$ & $67.8 \pm 17.1$ & 0.638 \\
\hline Gender (male) & $49(72.0)$ & $40(72.7)$ & 0.933 \\
\hline Current or exsmoker & $33(48.5)$ & $33(60.0)$ & 0.204 \\
\hline \multicolumn{4}{|l|}{ Co-morbidity } \\
\hline Chronic obstructive pulmonary disease & $28(4 \mid .2)$ & $25(56.4)$ & 0.633 \\
\hline Tumor & $13(33.8)$ & $6(10.9)$ & 0.210 \\
\hline Chronic heart failure & $10(14.7)$ & $15(27.3)$ & 0.085 \\
\hline Diabetes mellitus & $4(5.9)$ & $10(18.2)$ & $\underline{0.032}$ \\
\hline \multicolumn{4}{|l|}{ Laboratory tests } \\
\hline (I,3)- $\beta$-D-Glucan (normal: 60 pg/mL) & $116.8 \pm 250.9$ & $146.9 \pm 274.3$ & 0.481 \\
\hline CRP (normal: <10 mg/L) & $54.4 \pm 62.5$ & $61.3 \pm 42.0$ & 0.484 \\
\hline PCT (normal: $<0.05 \mu g / L)$ & $2.5 \pm 5.3$ & $2.8 \pm 7.3$ & 0.818 \\
\hline ESR (M: 2-2I, F: 2-25 mm/h) & $26.8 \pm 32.3$ & $27.9 \pm 33.0$ & 0.852 \\
\hline WBC (normal: 3.5-9.5 $\left.\times 10^{9} / L\right)$ & $10.7 \pm 5.6$ & $10.3 \pm 5.2$ & 0.339 \\
\hline Albumin (normal: $35-50 \mathrm{~g} / \mathrm{L}$ ) & $34.7 \pm 7.2$ & $29.8 \pm 5.3$ & $<\underline{0.001}$ \\
\hline \multicolumn{4}{|l|}{ Fungal culture } \\
\hline Positive fungal culture & $17(10.3)$ & $22(40.0)$ & 0.075 \\
\hline Aspergillus infection & $4(5.9)$ & $7(12.7)$ & 0.186 \\
\hline Candida infection & $13(19.1)$ & $14(25.5)$ & 0.398 \\
\hline \multicolumn{4}{|l|}{ Chest imaging findings } \\
\hline Cavity lesions & $8(11.8)$ & $3(5.5)$ & 0.222 \\
\hline Nodules & $22(32.4)$ & $8(16.4)$ & $\underline{0.022}$ \\
\hline Number with possible PFI & $39(57.4)$ & $24(43.6)$ & 0.130 \\
\hline \multicolumn{4}{|l|}{ Treatment and medical cost } \\
\hline Sequential antifungal therapy ${ }^{c}$ & $56(82.4)$ & $39(70.9)$ & 0.132 \\
\hline Voriconazole treatment & $24(35.3)$ & $28(50.9)$ & 0.081 \\
\hline Duration of antifungal therapy (days) & $12.2 \pm 19.9$ & $8.7 \pm 6.3$ & 0.159 \\
\hline Glucocorticoids & $30(44.1)$ & $29(52.7)$ & 0.341 \\
\hline Invasive operations & $5(7.4)$ & $25(45.6)$ & $\underline{0.000}$ \\
\hline Side effects of antifungal therapy & $5(7.4)$ & $7(12.7)$ & 0.317 \\
\hline Hospitalization cost (RMB) & $52,678 \pm 39,474$ & $81,745 \pm 71,4 \mid 8$ & $\underline{0.003}$ \\
\hline Hospitalization time (days) & $17.0 \pm 9.9$ & $16.0 \pm 12.2$ & 0.418 \\
\hline
\end{tabular}

Notes: ${ }^{a}$ Values are shown as mean \pm SD or $\mathrm{n}(\%)$. Underlined are values with statistical significance. Including fluconazole plus itraconzole, fluconazole plus voriconazole, fluconazole plus caspofungin or fluconazole plus amphotericin B. The dose and duration used: voriconazole ( $200 \mathrm{mg}$ twice a day for $2-165$ days), itraconazole (200 mg daily for 7-14 days), fluconazole (300 mg twice a day for I-30 days), caspofungin (50 mg daily for 4-14 days), or amphotericin B ( $10 \mathrm{mg}$ daily for $4-10$ days).

Abbreviations: CRP, C-reactive protein; ESR, erythrocyte sedimentation rate; F, female; M, male; PCT, procalcitonin; PFI, pulmonary fungal infection; WBC, white blood cell.

$72 \%$ males, 54\% smokers, and 35\% alcohol drinkers. All presented pulmonary symptoms, with the cough and expectoration being the most common (92\%), followed by dyspnea (20\%), chest pain (11\%), and hemoptysis (7\%). Fever was recorded in $38 \%$ of patients. Abnormal breath sounds were noted in $84 \%$ of patients, and oral or skin fungal infection was noted in $34 \%$ of patients. The majority (93\%) of the patients suffered at least one underlying disease, with COPD being the most common (45\%), followed by hypertension $(25 \%)$, heart disease (22\%), and various immune dysfunctions (37\%).

\section{Comparing possible and probable/proven PFls}

Among the 123 patients with PFIs, there were 63, 57, and three patients classified as possible, probable, and proven
PFIs, respectively. Due to the small sample size of the proven PFI group, it was combined into the probable PFI group forming the probable/proven PFI group. When comparing the clinical characteristics between these two groups (Table 5), a statistically significant difference was observed for the following characteristics, including a higher prevalence of hemoptysis ( 12 vs $2 \%, P=0.023$ ) and lung cavity lesions ( 15 vs $3 \%, P=0.021)$, a higher level of $1,3-\beta$-D-glucan $(254.0$ vs $12.3 \mathrm{pg} / \mathrm{mL}, P<0.0001)$, and a more frequent use of sequential antifungal therapy ( 30 vs $14 \%, P=0.035)$ in the probable/proven PFI group than in the possible PFI group. No statistically significant difference was observed for the remaining characteristics.

Of the 60 patients with probable/proven PFI, 44 (73\%) patients were positive in $1,3-\beta$-D-glucan test and $39(65 \%)$ 
Table 5 Comparison of clinical characteristics between possible PFI and probable/proven PFI

\begin{tabular}{|c|c|c|c|}
\hline Characteristics & Possible PFIa $(n=63)$ & Probable/proven PFIa $(n=60)$ & $P$-value ${ }^{b}$ \\
\hline Age (years) & $67.5 \pm 12.5$ & $57.5 \pm 17.7$ & 0.725 \\
\hline Gender (male) & $46(73.0)$ & $44(73.3)$ & 0.964 \\
\hline Current or exsmoker & $37(58.7)$ & $29(48.3)$ & 0.247 \\
\hline Current or former alcohol drinker & $25(39.7)$ & $18(30.0)$ & 0.260 \\
\hline \multicolumn{4}{|l|}{ Underlying conditions } \\
\hline Chronic obstructive pulmonary disease & $32(50.8)$ & $23(38.3)$ & 0.164 \\
\hline Diabetes mellitus & $8(12.7)$ & $6(10.0)$ & 0.637 \\
\hline Heart failure & $14(23.8)$ & II (18.3) & 0.592 \\
\hline Chronic renal disorder & $5(7.9)$ & $5(8.3)$ & 0.956 \\
\hline Malignancy & $12(19.0)$ & $8(13.3)$ & 0.257 \\
\hline \multicolumn{4}{|l|}{ Clinical manifestations } \\
\hline Hemoptysis & $\mathrm{I}(\mathrm{I} .6)$ & $7(11.7)$ & $\underline{0.023}$ \\
\hline Chest pain & $5(7.9)$ & $9(15.0)$ & $\overline{0.217}$ \\
\hline Cavity lesions & $2(3.2)$ & $9(15.0)$ & $\underline{0.021}$ \\
\hline \multicolumn{4}{|l|}{ Laboratory tests } \\
\hline CRP (normal: <10 mg/L) & $58.6 \pm 61.7$ & $57.0 \pm 45.5$ & 0.876 \\
\hline WBC (normal: $\left.3.5-9.5 \times 10^{9} / \mathrm{L}\right)$ & $10.8 \pm 6.6$ & $10.2 \pm 4.1$ & $0.56 \mathrm{I}$ \\
\hline $\operatorname{ESR}(\mathrm{M}: 2-2 \mathrm{I}, \mathrm{F}: 2-25 \mathrm{~mm} / \mathrm{h})$ & $25.9 \pm 25.9$ & $28.4 \pm 34.3$ & 0.678 \\
\hline PCT (normal: $<0.05 \mu g / L)$ & $3.2 \pm 8.5$ & $1.0 \pm 1.9$ & $\underline{0.0481}$ \\
\hline I,3-ß-D-Glucan (normal: <60 pg/mL) & $12.3 \pm 14.9$ & $254.0 \pm 331.8$ & $<\underline{0.0001}$ \\
\hline Albumin (normal: $35-50 \mathrm{~g} / \mathrm{L}$ ) & $31.7 \pm 6.2$ & $32.1 \pm 7.2$ & 0.776 \\
\hline \multicolumn{4}{|l|}{ Treatment outcome and cost } \\
\hline Duration of antifungal treatment (days) & $8.7 \pm 5.7$ & $|3.1 \pm 2| .7$ & 0.125 \\
\hline Sequential antifungal therapy & $9(14.3)$ & $18(30.0)$ & $\underline{0.035}$ \\
\hline Favorable response & $40(63.5)$ & $29(48.3)$ & 0.090 \\
\hline Death & $12(19.0)$ & $17(28.3)$ & $\overline{0.225}$ \\
\hline Hospitalization costs (RMB) & $58,465 \pm 52,292$ & $73,247 \pm 62,736$ & 0.156 \\
\hline Hospitalization duration (days) & $15.8 \pm 10.3$ & $|7.4 \pm 1| .7$ & 0.414 \\
\hline
\end{tabular}

Notes: a $V$ alues are shown as mean \pm SD or $\mathrm{n}(\%)$. 'Underlined are values with statistical significance.

Abbreviations: CRP, C-reactive protein; ESR, erythrocyte sedimentation rate; F, female; PCT, procalcitonin; M, male; PFI, pulmonary fungal infection; WBC, white blood cell.

patients were positive in fungal culture and/or microscopic examination (Table 3). A total of seven different fungal species were identified, with Candida albicans being the most prevalent species (20/39 or $51 \%)$, followed by Aspergillus fumigatus (10/39 or 26\%). Other fungal species were rare $(<8 \%$ each). Based on in vitro drug sensitivity test, no apparent drug resistance was noted for any of these fungal species. There was no significant difference in any demographic and clinical characteristics between Candida- and Aspergillus-infected patients except that the latter group had a younger age, a higher prevalence of lung cavity lesions, and a higher white blood cell (WBC) count $(P<0.05$ each) (Table S1).

\section{Comparisons of treatment responses}

All of the 123 patients with PFIs were treated with antifungal agents during hospitalization, with $68(55 \%)$ of them showing favorable responses and the remaining 55 (45\%) showing unfavorable responses (Table 4). Compared with patients with favorable responses, patients with unfavorable responses had a higher prevalence of diabetes mellitus $(P=0.032)$, a lower albumin level $(P<0.001)$, a lower prevalence of lung nodules in radiography $(P=0.022)$, a higher rate of receiving invasive operation $(P=0.000)$, and a higher medical cost during hospitalization $(P=0.003)$. No statistically significant difference was observed for other categories compared.

\section{Prognostic factors associated with mortality}

Twenty-nine (24\%) of the 123 PFI patients died during the hospital stay. As per multivariate logistic regression analysis (Table 6), hypoalbuminemia (odds ratio [OR]: 3.936, 95\% confidence interval [CI]: $1.325-11.696, P=0.014)$, invasive operation (OR: $5.736,95 \%$ CI: $2.008-16.389, P=0.001$ ), and older ages ( $\geq 60$ years, OR: $5.739,95 \%$ CI: 1.049-31.401, $P=0.044)$ were independent prognostic factors associated with mortality. Other risk factors surveyed were not associated with mortality $(P>0.05$ each). 
Table 6 Regression analysis of prognostic factors associated with mortality for 29 PFI patients

\begin{tabular}{|c|c|c|}
\hline Risk factors & OR (95\% CI) & $P$-value ${ }^{a}$ \\
\hline Age $\geq 60$ years & $5.739(1.049-31.401)$ & $\underline{0.044}$ \\
\hline Gender (male) & $0.440(0.137-I .4 I I)$ & 0.167 \\
\hline Leukopenia $\left(<4 \times 10^{9} / \mathrm{L}\right)$ & $0.000(0.000)$ & 0.999 \\
\hline Hypoalbuminemia (<30 g/L) & $3.936(1.325-11.696)$ & $\underline{0.014}$ \\
\hline Invasive operations & $5.736(2.008-16.389)$ & $\underline{0.001}$ \\
\hline Use of immunosuppressive agents & $2.040(0.222-18.750)$ & 0.529 \\
\hline Use of broad-spectrum antibiotics $>2$ weeks & $0.873(0.385-1.980)$ & 0.745 \\
\hline Use of glucocorticoids $>2$ weeks & $1.403(0.308-6.399)$ & 0.662 \\
\hline Prolonged hospitalization ( $>10$ days) & $0.908(0.210-3.924)$ & 0.897 \\
\hline Diabetes mellitus & $3.348(0.74 I-15.125)$ & 0.116 \\
\hline Hematological or solid organ malignancy & $0.673(0.131-3.452)$ & 0.635 \\
\hline
\end{tabular}

Note: anderlined are values with statistical significance.

Abbreviations: $\mathrm{Cl}$, confidence interval; OR, odds ratio; PFI, pulmonary fungal infection.

\section{Discussion}

To better understand the clinical characteristics and prognostic factors of PFIs, we retrospectively investigated a panel of 123 PFI patients with diverse demographic and risk factors and clinical and laboratory profiles.

We evaluated the prevalence of known potential risk factors for PFIs (Table 1) and found that the most prevalent risk factor is prolonged hospitalization (74\%). This observation is consistent with previous studies of fungal infection in the lungs ${ }^{15}$ and other organs. ${ }^{16} \mathrm{It}$ is conceivable that prolonged hospitalization is likely to increase the chances of exposure to fungal pathogens, which are rich in the hospital environment. ${ }^{17}$ Nevertheless, prolonged hospitalization is often not an independent risk factors and it is usually associated with chronic or severe disorders as presented in Table 1. Other common risk factors that we observed in this study are similar to previous reports. ${ }^{10,18-21}$ Of note, neutrophil dysfunction was not noted in any of the PFI patients involved in this study, though it has been considered as one of the most significant traditional risk factors for PFI. ${ }^{1,8,22}$

The main clinical characteristics of the 123 PFI patients in this study include a high prevalence in elderly patients (79\%) and males (72\%), along with symptoms dominated by cough and expectoration (92\%), fever (38\%), dyspnea (20\%), and chest pain (11\%). The high prevalence in males is in line with previous studies (52-71\%) from China ${ }^{5,6}$ and other countries. ${ }^{21,23,24}$ However, the mean age of the patients in our study (67 years) appears to be older than that reported in China (45 or 53 years). ${ }^{5,6}$ In addition, we observed a much higher prevalence of PFI in the elderly ( $\geq 60$ years) than in adults ( $<60$ years). It is likely that the elders have weaker immune functions and/or are accompanied with other disorders, thus leading to a greater chance to be infected by fungal pathogens.

While the prevalence of most of the symptoms we observed appears to be similar to that reported previously, we observed a lower prevalence of hemoptysis (7\%) and a higher prevalence of abnormal breath sounds (84\%) compared to previous reports from China, in which hemoptysis and abnormal breath sounds were observed in $32-37 \%$ and $18-63 \%$ PFI patients, respectively. ${ }^{4,6}$ Despite the low prevalence of hemoptysis in our study, we observed a significantly higher prevalence of hemoptysis in probable/proven PFI patients than in possible PFI patients ( 12 vs $2 \%, P=0.023$ ), which is consistent with the higher prevalence of lung cavity lesions in probable/proven PFI patients than in possible PFI patients (15 vs $3 \%, P=0.021$, Table 5 ). Of note, oral or skin fungal infection was observed in $34 \%$ of patients in this study, which has been rarely reported previously, with one study showing a lower incidence of $16 \%$ in PFI patients with hematological malignancies. ${ }^{15}$ Further studies are needed to verify whether this observation is causal or coincidental. The most common underlying disease for the PFI patients in this study is COPD, with a prevalence of $45 \%$ (Table 2), which appears to be much higher than the previously reported rate of $0-19 \%$ from China ${ }^{5,6,15}$ and $1.3-17 \%$ from other countries. ${ }^{18,19,24-26}$ While it remains unclear why PFIs are highly prevalent in COPD, it has been hypothesized that COPD can increase susceptibility to fungal infection due to lung structure changes resulting from frequent bacterial infections or other underlying diseases, frequent hospitalization, and repeated use of systemic corticosteroids and broad-spectrum antibiotics. ${ }^{25}$

Clinical manifestations and radiological findings of PFI are nonspecific and highly variable; definite diagnosis relies on positive microscopic or culture findings. ${ }^{27}$ In the present study, $39(65 \%)$ of the 60 patients with probable/proven PFI were positive in fungal culture and microscopic examination (Table 3). Strikingly, most (67\%) of the 39 fungal-positive patients were infected with Candida spp; only $28 \%$ of them were infected with Aspergillus spp (28\%); and 5\% of them were infected with 
Cryptococcus spp. These findings are consistent with the report of $\mathrm{Hu}$ et $\mathrm{al}^{15}$ from China but contradict the observation of the dominance of A. fumigatus infection among PFI patients in many other studies in China ${ }^{5,6}$ and around the world. ${ }^{8-10}$ Further comparison showed that Candida-infected patients had an older age, a lower rate of lung cavity lesions, and a lower WBC count compared to Aspergillus-infected patients. Nonetheless, these observations await further confirmation using a larger number of patients with diverse geographic origins.

In the present study, all PFI patients were treated with antifungal agents; 68 (55\%) of them showed favorable responses (Table 4). Favorable responses were significantly associated with a higher level of serum albumin, a higher prevalence of lung nodules, a lower rate of invasive operations, a lower prevalence of diabetes mellitus, and a lower medical cost during hospitalization (Table 4). The treatment response rate in our study appears to be higher than the favorable response rate of $29 \%$ reported in $\mathrm{China}^{6}$ but similar to the rate of $38-68 \%$ reported in other countries. ${ }^{28-30}$ The relatively high rate of favorable responses in our study could be explained partially by the absence of apparent drug resistance in in vitro susceptibility test. Although previous studies have suggested that sequential treatment with different antifungal agents can improve responses, ${ }^{6}$ we did not observe a positive correlation of sequential treatment with favorable responses (Table 4). Nevertheless, treatment response rates may vary based on the patient population, treatment regimens, and the definition of responses. Clearly, further well-controlled studies are needed to draw a reliable conclusion.

The mortality rate observed in this study is $24 \%(29 / 123)$. Multivariate regression analysis showed that hypoalbuminemia and invasive operations were independent prognostic factors associated with mortality (Table 6), which is consistent with the positive associations of these two factors with unfavorable treatment responses (Table 4). This mortality rate is similar to the rate of $21-39 \%$ reported previously in China and around the world. ${ }^{30-34}$ The observation of hypoalbuminemia as an independent prognostic factor for mortality is in agreement with previous studies. ${ }^{15,33}$ The association of hypoalbuminemia with poor treatment outcomes could be explained by its impact on pharmacokinetics of antifungal drugs shown in a recent study suggesting that hypoalbuminemia can increase unbound voriconazole concentrations in plasma, thereby increasing drug elimination and attenuating its therapeutic effects. ${ }^{35}$ These findings suggest a need to correct or seek and treat the causes of hypoalbuminemia in patients with PFIs.

The observation of invasive operation as an independent risk factor for the mortality of PFIs in this study is intrigu- ing as this has not been reported previously. In our case series, $30(24 \%)$ of the 123 PFI patients received invasive operation (primarily urinary catheterization, Table 1) and it was significantly more frequently involved in patients with unfavorable treatment responses than in patients with favorable responses ( 45.6 vs $7.4 \%, P=0.000$, Table 4 ). The strong association of invasive operation with both poor treatment responses and mortality supports it to be an important risk factor in the development of PFIs. Certainly, further investigation is warranted to validate this association by studying more patients from different regions and to determine its underlying mechanisms.

There are some limitations in this study. First, it was done in only one hospital without a large number of patients and, therefore, whether the clinical findings can be extrapolated to PFI cases in other regions is unclear. It is certainly desirable to extend this study to other geographical regions of our country. Second, none of the fungal species identified were confirmed by molecular methods such as polymerase chain reaction and sequence analysis and, thus, the possibility of misidentification cannot be completely ruled out. Third, treatment outcomes were evaluated based on the responses of patients after a variable duration of treatment and follow-up. In addition, the severity of patients prior to treatment was not scored, which may introduce bias in analysis of treatment outcomes.

\section{Conclusion}

We retrospectively investigated a case series of PFI patients with diverse demographic factors and medical conditions and demonstrated their clinical characteristics and treatment outcomes. The main novelty of this study is the demonstration, for the first time, that COPD is the most common underlying disease $(45 \%)$ in PFI patients and that invasive operation is significantly associated with poor treatment responses and mortality in PFI patients. High index of suspicion of PFIs in patients with COPD and patients receiving invasive operation is necessary for timely, appropriate clinical management of these patients.

\section{Acknowledgments}

All authors have reported that no personal or financial support was received for this study. All clinical work was performed in the First Affiliated Hospital of Chongqing Medical University, Chongqing, China.

\section{Author contributions}

LP is the guarantor of this article. LP and ZX contributed to the conception and design and to the acquisition, analysis, and interpretations of data. $\mathrm{RL}, \mathrm{ZH}$, and LM participated 
in the analysis and interpretations of data. LP, ZX, and LM drafted and edited the submitted article. All authors provided final approval of the version to be published. All authors contributed toward data analysis, drafting and critically revising the paper and agree to be accountable for all aspects of the work.

\section{Disclosure}

The authors report no conflicts of interest in this work.

\section{References}

1. Limper AH. The changing spectrum of fungal infections in pulmonary and critical care practice: clinical approach to diagnosis. Proc Am Thorac Soc. 2010;7(3):163-168.

2. Maschmeyer G, Haas A, Cornely OA. Invasive aspergillosis: epidemiology, diagnosis and management in immunocompromised patients. Drugs. 2007;67(11):1567-1601.

3. Arvanitis M, Mylonakis E. Diagnosis of invasive aspergillosis: recent developments and ongoing challenges. Eur J Clin Invest. 2015;45(6): 646-652.

4. Chen KY, Ko SC, Hsueh PR, Luh KT, Yang PC. Pulmonary fungal infection: emphasis on microbiological spectra, patient outcome, and prognostic factors. Chest. 2001;120(1):177-184.

5. Liu YN, She DY, Sun TY, et al. [A multicentre retrospective study of pulmonary mycosis clinically proven from 1998 to 2007]. Zhonghua Jie He He Hu Xi Za Zhi. 2011;34(2):86-90.

6. Luo BL, Zhang LM, Hu CP, Xiong Z. Clinical analysis of 68 patients with pulmonary mycosis in China. Multidiscip Respir Med. 2011;6(5):278-283.

7. De Pauw B, Walsh TJ, Donnelly JP, et al. Revised definitions of invasive fungal disease from the European Organization for Research and Treatment of Cancer/Invasive Fungal Infections Cooperative Group and the National Institute of Allergy and Infectious Diseases Mycoses Study Group (EORTC/MSG) Consensus group. Clin Infect Dis. 2008;46(12):1813-1821.

8. Gerson SL, Talbot GH, Hurwitz S, Strom BL, Lusk EJ, Cassileth PA. Prolonged granulocytopenia: the major risk factor for invasive pulmonary aspergillosis in patients with acute leukemia. Ann Intern Med. 1984;100(3):345-351.

9. Gonzalez CE, Venzon D, Lee S, Mueller BU, Pizzo PA, Walsh TJ. Risk factors for fungemia in children infected with human immunodeficiency virus: a case-control study. Clin Infect Dis. 1996;23(3):515-521.

10. Pound MW, Drew RH, Perfect JR. Recent advances in the epidemiology, prevention, diagnosis, and treatment of fungal pneumonia. Curr Opin Infect Dis. 2002;15(2):183-194.

11. Singh G, Pitoyo CW, Aditianingsih D, Rumende CM. Risk factors for early invasive fungal disease in critically ill patients. Indian J Crit Care Med. 2016;20(11):633-639.

12. Villarroel M, Aviles CL, Silva P, et al. Risk factors associated with invasive fungal disease in children with cancer and febrile neutropenia: a prospective multicenter evaluation. Pediatr Infect Dis J. 2010;29(9):816-821.

13. Greene RE, Schlamm HT, Oestmann JW, et al. Imaging findings in acute invasive pulmonary aspergillosis: clinical significance of the halo sign. Clin Infect Dis. 2007;44(3):373-379.

14. Jhun BW, Jeon K, Eom JS, et al. Clinical characteristics and treatment outcomes of chronic pulmonary aspergillosis. Med Mycol. 2013;51(8):811-817.

15. Hu R, Jiang XY, Wu Y. Risk factors for invasive pulmonary fungal infection in patients with hematological malignancies not receiving hematopoietic stem cell transplant. Neoplasma. 2012;59(6): 669-675.
16. Kochhar R, Ahammed SK, Chakrabarti A, et al. Prevalence and outcome of fungal infection in patients with severe acute pancreatitis. $J$ Gastroenterol Hepatol. 2009;24(5):743-747.

17. Tong X, Xu H, Zou L, et al. High diversity of airborne fungi in the hospital environment as revealed by meta-sequencing-based microbiome analysis. Sci Rep. 2017;7:39606.

18. Alsalman J, Zaid T, Makhlooq M, et al. A retrospective study of the epidemiology and clinical manifestation of invasive aspergillosis in a major tertiary care hospital in Bahrain. J Infect Public Health. 2017;10(1):49-58.

19. Ginea J, Torres-Narbona M, Gijon P, et al. Pulmonary aspergillosis in patients with chronic obstructive pulmonary disease: incidence, risk factors, and outcome. Clin Microbiol Infect. 2010;16(7):870-877.

20. Taccone FS, Van den Abeele AM, Bulpa P, et al; AspICU Study Investigators. Epidemiology of invasive aspergillosis in critically ill patients: clinical presentation, underlying conditions, and outcomes. Crit Care. 2015;19:7.

21. Lanternier F, Dannaoui E, Morizot G, et al; French Mycosis Study Group. A global analysis of mucormycosis in France: the RetroZygo study (2005-2007). Clin Infect Dis. 2012;54(suppl 1):S35-S43.

22. Trof RJ, Beishuizen A, Debets-Ossenkopp YJ, Girbes AR, Groeneveld AB. Management of invasive pulmonary aspergillosis in non-neutropenic critically ill patients. Intensive Care Med. 2007;33(10):1694-1703.

23. Gamaletsou MN, Rammaert B, Bueno MA, et al. Aspergillus osteomyelitis: epidemiology, clinical manifestations, management, and outcome. J Infect. 2014;68(5):478-493.

24. Corzo-Leon DE, Armstrong-James D, Denning DW. Burden of serious fungal infections in Mexico. Mycoses. 2015;58(suppl 5):34-44.

25. Ader F, Nseir S, Le Berre R, et al. Invasive pulmonary aspergillosis in chronic obstructive pulmonary disease: an emerging fungal pathogen. Clin Microbiol Infect. 2005;11(6):427-429.

26. Khelafi R, Aissanou A, Tarsift S, Skander F. [Epidemiology of chronic obstructive pulmonary disease in Algiers]. Rev Mal Respir. 2011;28(1):32-40.

27. Arvanitis M, Ziakas PD, Zacharioudakis IM, Zervou FN, Caliendo AM, Mylonakis E. PCR in diagnosis of invasive aspergillosis: a meta-analysis of diagnostic performance. J Clin Microbiol. 2014;52(10):3731-3742.

28. Betts R, Glasmacher A, Maertens J, et al. Efficacy of caspofungin against invasive Candida or invasive Aspergillus infections in neutropenic patients. Cancer. 2006;106(2):466-473.

29. Cornely OA, Gachot B, Akan H, et al; EORTC Infectious Diseases Group. Epidemiology and outcome of fungemia in a cancer Cohort of the Infectious Diseases Group (IDG) of the European Organization for Research and Treatment of Cancer (EORTC 65031). Clin Infect Dis. 2015;61(3):324-331.

30. Costa-de-Oliveira S, Pina-Vaz C, Mendonca D, Gonçalves Rodrigues A. A first Portuguese epidemiological survey of fungaemia in a university hospital. Eur J Clin Microbiol Infect Dis. 2008;27(5):365-374.

31. Liu ZY, Sheng RY, Li XL, Li TS, Wang AX. [Nosocomial fungal infections, analysis of 149 cases]. Zhonghua Yi Xue Za Zhi. 2003;83(5):399-402. Chinese.

32. Neofytos D, Horn D, Anaissie E, et al. Epidemiology and outcome of invasive fungal infection in adult hematopoietic stem cell transplant recipients: analysis of Multicenter Prospective Antifungal Therapy (PATH) Alliance registry. Clin Infect Dis. 2009;48(3):265-273.

33. Yan X, Zong F, Kong H, et al. Pulmonary fungal diseases in immunocompetent hosts: a single-center retrospective analysis of 35 subjects. Mycopathologia. 2016;181(7-8):513-521.

34. Zhang L, Liu ZY, Xu YC, Li TS, Wang Y, Wang P. [The clinical characteristics of 70 cases of fungemia]. Zhonghua Nei Ke Za Zhi. 2012;51(12): 952-956. Chinese.

35. Vanstraelen K, Wauters J, Vercammen I, et al. Impact of hypoalbuminemia on voriconazole pharmacokinetics in critically ill adult patients. Antimicrob Agents Chemother. 2014;58(11):6782-6789.

36. World Health Organization. Technical consultation on indicators of adolescent health, WHO, Geneva, Switzerland, 30 September - 1 October 2014: global reference list of health indicators for adolescents (aged 10-19 years). Geneva: World Health Organization; 2016. Available from: http:// apps.who.int/iris/handle/10665/204625. Accessed February 2, 2018. 


\section{Supplementary material}

Table SI Comparison of clinical characteristics between Candida and Aspergillus infection

\begin{tabular}{|c|c|c|c|}
\hline Characteristics & Candida spp $^{a}(n=26)$ & Aspergillus spp ${ }^{a}(n=I I)$ & $P$-value ${ }^{b}$ \\
\hline Age (years) & $72.6 \pm 12.7$ & $57.9 \pm 22.9$ & $\underline{0.022}$ \\
\hline Gender (male) & $22(84.6)$ & $8(72.7)$ & 0.399 \\
\hline Current or exsmoker & I5 (57.7) & $5(4 I .7)$ & 0.495 \\
\hline Alcohol drinker & $10(38.5)$ & $4(36.7)$ & 0.904 \\
\hline \multicolumn{4}{|l|}{ Underlying diseases/risk factors } \\
\hline Chronic obstructive pulmonary disease & $12(46.2)$ & $6(54.5)$ & 0641 \\
\hline HIV infection & $2(7.7)$ & $0(0)$ & 0.344 \\
\hline Invasive operation & $5(19.2)$ & $3(27.3)$ & 0.557 \\
\hline Diabetes mellitus & $4(15.4)$ & $0(0)$ & 0.168 \\
\hline Skin/oral fungal infection & $8(30.8)$ & $3(27.3)$ & 0.833 \\
\hline \multicolumn{4}{|l|}{ Clinical manifestations } \\
\hline Hemoptysis & $3(11.5)$ & $0(0)$ & 0.240 \\
\hline Fever & $12(46.2)$ & $8(72.7)$ & 0.138 \\
\hline Nodules & $9(34.6)$ & $5(45.5)$ & 0.534 \\
\hline Cavity lesions & I (3.9) & $4(36.7)$ & $\underline{0.008}$ \\
\hline \multicolumn{4}{|l|}{ Laboratory tests } \\
\hline I,3- $\beta$-D-Glucan (normal: $<60 \mathrm{pg} / \mathrm{mL}$ ) & $167.1 \pm 282.1$ & $150.1 \pm 288.3$ & 0.674 \\
\hline CRP (normal: < 10 mg/L) & $77.1 \pm 49.5$ & $66.8 \pm 35.2$ & 0.726 \\
\hline PCT (normal: $<0.05 \mu g / L)$ & $I . I \pm 2 . I$ & $0.7 \pm 0.9$ & 1.000 \\
\hline ESR (M: 2-2I, F: 2-25 mm/h) & $27.7 \pm 31.8$ & $34.5 \pm 38.3$ & 0.503 \\
\hline WBC (normal: $3.5-9.5 \times 109 / L)$ & $9.6 \pm 3.8$ & $13.6 \pm 3.5$ & $\underline{0.013}$ \\
\hline Albumin (normal: $35-50 \mathrm{~g} / \mathrm{L}$ ) & $32.3 \pm 8.5$ & $30.2 \pm 6.7$ & 0.368 \\
\hline \multicolumn{4}{|l|}{ Treatment outcome and cost } \\
\hline Death & II (42.3) & $4(36.7)$ & 0.736 \\
\hline Favorable response & $10(38.5)$ & $5(45.5)$ & 0.692 \\
\hline Hospitalization costs (RMB) & $79,170 \pm 62,240$ & $92,940 \pm 95,940$ & 0.907 \\
\hline Hospitalization duration (days) & $16.9 \pm 11.2$ & $23.9 \pm 17.6$ & 0.117 \\
\hline
\end{tabular}

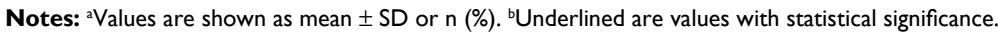

Abbreviations: CRP, C-reactive protein; ESR, erythrocyte sedimentation rate; F, female; HIV, human immunodeficiency virus; M, male; PCT, procalcitonin; WBC, white blood cell.

\section{Publish your work in this journal}

Infection and Drug Resistance is an international, peer-reviewed openaccess journal that focuses on the optimal treatment of infection (bacterial, fungal and viral) and the development and institution of preventive strategies to minimize the development and spread of resistance. The journal is specifically concerned with the epidemiology of antibiotic

\section{Dovepress}

resistance and the mechanisms of resistance development and diffusion in both hospitals and the community. The manuscript management system is completely online and includes a very quick and fair peerreview system, which is all easy to use. Visit http://www.dovepress.com/ testimonials.php to read real quotes from published authors.

Submit your manuscript here: https://www.dovepress.com/infection-and-drug-resistance-journal 